\title{
BMJ Open Electronic patient reported outcomes to support care of patients with traumatic brain injury: PRiORiTy study qualitative protocol
}

\author{
Ameeta Retzer, ${ }^{1,2}$ Grace M Turner, ${ }^{1,2}$ Anita Slade, ${ }^{1,2,3}$ Derek Kyte, ${ }^{1,3}$ \\ Christel McMullan, ${ }^{1,2}$ Laura Jones, ${ }^{1}$ Antonio Belli, ${ }^{2}$ Melanie Calvert ${ }^{1,2,3}$
}

To cite: Retzer A, Turner GM, Slade A, et al. Electronic patient reported outcomes to support care of patients with traumatic brain injury: PRiORiTy study qualitative protocol. BMJ Open 2019;9:e024617. doi:10.1136/ bmjopen-2018-024617

- Prepublication history and additional material for this paper are available online. To view these files, please visit the journal online (http://dx.doi. org/10.1136/bmjopen-2018024617).

Received 7 June 2018 Revised 11 September 2018 Accepted 7 December 2018

\section{Check for updates}

(C) Author(s) (or their employer(s)) 2019. Re-use permitted under CC BY-NC. No commercial re-use. See rights and permissions. Published by BMJ.

${ }^{1}$ Centre for Patient Reported Outcomes Research, Institute of Applied Health Research, University of Birmingham, Birmingham, UK

${ }^{2}$ National Institute for Health Research Surgical Reconstruction and Microbiology Research Centre, Queen

Elizabeth Hospital Birmingham, Birmingham, UK

${ }^{3} \mathrm{NIHR}$ Birmingham Biomedical Research Centre, University Hospitals Birmingham NHS Foundation Trust and University of Birmingham, Birmingham

Correspondence to

Dr Grace M Turner;

g.turner.1@bham.ac.uk

\section{ABSTRACT}

Introduction Traumatic brain injury (TBI) represents a major health and socioeconomic problem internationally. The expansive nature of injuries results in a heterogeneous population. The degree and type of long-term impacts following TBI and improvement following injury are highly variable. The use of electronic Patient Reported Outcomes Measures (ePROMs) could help identify residual impacts of TBI and support patient management and care. The Patient Reported Outcomes Research in Trauma study is a qualitative study exploring the long-term symptoms and impacts that are experienced by those with TBI and the potential utility of an ePROM platform to collect real-time information on patient symptoms and quality of life to inform treatment and identify support needs.

Methods and analysis Semi-structured telephone and face-to-face interviews will be conducted with approximately 30-40 individuals recruited from five groups: (1) people with TBI; (2) carers and relatives of individuals with TBI; (3) TBI healthcare professionals; (4) researchers and (5) third sector staff members and volunteers working with those with TBI. Data will be analysed using directed thematic analysis employing an iterative coding frame that will be modified as analysis progresses. Intercoder triangulation will be employed to enhance credibility.

Ethics and dissemination This study was approved by the West Midlands-Black Country Research Ethics Committee (Ref: 18/WM/0033). Findings will be disseminated via conference presentations, peer-reviewed journals, social media (@CPROR_UoB; http://www. birmingham.ac.uk/cpror) and the National Institute for Health Research Surgical Reconstruction and Microbiology Research Centre.

\section{INTRODUCTION}

Head injury, which encompasses all types of damage to the head, is the most common cause of death and disability among people aged between 1 and 40 years in the UK. ${ }^{1}$ Each year 1.4 million people attend accident and emergency departments in England and Wales due to recent head injury. ${ }^{1}$ Traumatic brain injury (TBI) is caused by trauma to the

\section{Strengths and limitations of this study}

- Use of electronic Patient Reported Outcomes Measures (ePROMs) could help identify residual impacts of traumatic brain injury (TBI) and support patient management and care. This qualitative study explores long-term symptoms and impacts of TBI and the potential utility of an ePROM platform to collect information on patients' symptoms and quality of life to inform treatment and identify support needs.

- The semistructured interview format ensures a replicable process while allowing sufficient flexibility to explore new and emerging concepts.

- This study will draw on the experience of a wide range of stakeholders with the aim of capturing the most diverse possible range of perspectives to inform the development of an ePROM system.

- Patient participants receiving inpatient and outpatient care will be drawn from one site (Queen Elizabeth Hospital Birmingham) which may limit the transferability of our conclusions to that locality. However, this will be mediated by recruitment of those receiving support from regional and national third sector organisations.

- Our sample may be biased by the inclusion of only participants who are assessed to have capacity to consent. As such, we will be unable to capture the perspectives and experiences of those who do not meet these criteria. However, this may be an avenue for future research.

head, and includes the effects on the brain of other possible complications of the injury, notably hypoxaemia and hypotension, and intracerebral haematoma. ${ }^{2}$ TBI represents a major health and socioeconomic problem internationally ${ }^{3}$ and the expansive nature of injuries, including those caused by falls, involvement in traffic incidents, interpersonal violence, conflict and terrorism, results in a heterogeneous population that challenges current concepts of classification and research methodology. 
The degree of long-term impacts following TBI is highly variable, ranging from no measurable impact to severe impairment as indicated by neuroimaging and neuropsychological assessment, and within these, improvement following injury is also subject to great variation. ${ }^{5}$ The types of long-term effect are also wide ranging. ${ }^{6-8}$ Existing qualitative research shows how those living with the effects of TBI experience complex several long-term impacts, including exhibiting challenging behaviours, ${ }^{9}$ fatigue, ${ }^{10}$ mental health and cognition problems, ${ }^{11-14}$ within the wider context of accepting and adapting to post-injury circumstances. ${ }^{1516}$ Carers and relatives of those who have experienced TBI also report experiencing their own unique set of challenges and quality of life related effects relating to these. ${ }^{17-19}$

Advances in critical care, imaging and the reorganisation of trauma health systems have led to improved recognition and treatment of these complications, leading to significant reduction in deaths and disability caused by TBI. ${ }^{20}$ Despite this, research indicates the needs and experiences of people following TBI warrants further in-depth qualitative study so that more effective care and support may be provided..$^{21} 22$

One method of systematically capturing patients' own health perspectives is through the use of Patient Reported Outcome Measures (PROMs). These are questionnaires completed by patients to measure their own estimation of their health using domains such as symptoms, mobility, mental health and social function. ${ }^{23}$ PROMs may be a useful adjunct to clinical outcomes, which may underestimate the impact of a condition. ${ }^{24}$ Patients' own reports better reflect their daily health status whereas clinicians' assessments better predict unfavourable clinical events; when used in combination, both forms of data provide valuable insight into patients' outcomes and experiences. ${ }^{25}$

PROM data may be collected as part of routine clinical practice to aid communication; support shared decision-making; improve patient activation and engagement in treatment decisions; improve symptom assessment and management; reduce hospital admissions and improve health-related quality of life and quality adjusted survival. ${ }^{26}$ For individuals, they may inform patient treatment choices; facilitate cooperation between healthcare teams to provide personalised care and identify those most in need of intervention. At a systems level, PROM data may be used for audit and quality assurance; provide long-term safety and effectiveness data for treatments and inform pay-for-performance initiatives, prognostic modelling and health policy. ${ }^{27}$

PROMS are traditionally collected through the use of paper questionnaires; however, the introduction of electronic PROMs (ePROMs) has important advantages in comparison. The use of ePROMs reduces administrative burden and secondary data entry errors; are conducive to collecting real-time data; may facilitate remote monitoring and response by the clinical team; and may reduce patient burden through computer adaptive tests. ${ }^{28}$
Lived experience of TBI from a patient and carer perspective is well documented in the literature ${ }^{29}$; however, there is a lack of qualitative exploration with TBI healthcare professionals and researchers. Although previous research has provided recommendations on core outcome measures for TBI, ${ }^{30-34}$ patients and carers/ relatives have not been included in the selection process and their experiences and opinions of completing PROMs for clinical and/or research purposes have not been explored. Recommended outcome measures have been selected in the context of clinical research; however, it is important to ensure outcome measures for research align with clinical and rehabilitation priorities to avoid duplicate and competing data collection. The Patient Reported Outcomes Research in Trauma (PRiORiTy) study will explore outcomes of importance from the perspective of patients with TBI and their carers/relatives; healthcare providers, including the third/voluntarysector; and researchers, and investigate the potential utility of an ePROM platform to collect real-time information on patient symptoms and quality of life to inform treatment and identify support needs. The PRiORiTy study is nested within a larger programme of research aiming to develop and assess the feasibility of an ePROM pathway for inclusion within routine clinical care and TBI research.

This paper provides a summary of the qualitative study protocol in which semi-structured interviews will be conducted to inform the development of an ePROM system with: people with TBI; their relatives and carers; and TBI healthcare professionals, researchers and third sector/voluntary workers.

\section{OBJECTIVES}

Semi-structured telephone or face-to-face interviews will be conducted to explore the perspectives and experience of key stakeholders in relation to:

1. The key outcomes of importance to each participant group.

2. Patients' and carers' experiences of living with TBI and the impact of living with long-term symptoms.

3. Common symptoms that are experienced across the TBI population.

4. Healthcare professionals' and third sector/voluntary workers' experience of caring for those with TBI and managing their symptoms.

5. Current use and experience of PROMs by participants and their use in routine care and research.

6. The necessary features and functions of an ePROM, and where it may be situated.

\section{METHODS AND ANALYSIS}

\section{Participants and setting}

Inclusion criteria are that participants:

- Must have experience of TBI, within a professional capacity or through lived experience. 
- Are aged 18 years and over.

- Are able to converse in everyday English.

- Have capacity to provide informed consent to participate in the study.

Interviewees will be sampled from five groups: (1) people who have experienced TBI; (2) relatives and carers of individuals with TBI; (3) TBI healthcare professionals; (4) researchers and (5) third sector staff members and volunteers. Based on the experience of the research team, it is anticipated that the recruitment of approximately $6-8$ individuals from each group will be required to reach analytical saturation. Thus, approximately 30-40 participants will be required. Data collection will continue until the research team judge that the data and sample have sufficient depth and breadth to address the research questions.

Group 1 will be identified using three strategies intended to maximise sample diversity and will include inpatients, outpatients and those receiving support from third sector organisations. Diagnosis of TBI will be confirmed by a consultant neurosurgeon; however, we will not collect participants' clinical data. Inpatients will be identified by a research nurse from Queen Elizabeth Hospital Birmingham (QEHB) in accordance with the eligibility criteria. Outpatients will be those attending a neurosurgical follow-up clinic at QEHB, identified by research nurse and clinical members of the research team in accordance with the eligibility criteria. Potential inpatient and outpatient participants will be provided with information and the participant information sheet (PIS) by the research nurse. If they express an interest in participating in the PRiORiTy study, the research nurse will take written consent allowing for their contact details to be shared with the research team so that an interview may be arranged. Those receiving support from third sector organisations will be contacted through snowballing and circulation of information on social media and third sector support organisations.

Group 2 will be contacted through the circulation of information on social media and support groups and charities. Groups 3 and 4 will be identified and approached by research nurses and clinical members of the research team at QEHB, in addition to circulation of information via the National Institute for Health Research Surgical Reconstruction and Microbiology Research Centre (NIHR SRMRC) national networks, known contacts of the research team and snowballing. Group 5 will be contacted through the circulation of information to third sector organisations and snowballing. All participants will be based in the UK.

Interviews will take place via telephone, Skype or faceto-face at the University of Birmingham, QEHB, participants' homes or a neutral location, depending on the participants' preference.

\section{Data collection}

Potential participants will be provided with a brief outline of the research aims, PIS and details of how to register interest. Those interested in participating and who consented to the contacted will be contacted by a member of the research team and will be given further opportunity to ask questions before deciding whether to take part in an interview. An interview date will be set for those wishing to participate. Due to logistical and practical reasons, research nurses will arrange the time and location at QEHB for group 1 participants receiving inpatient care through liaison with the research team. All other participants will be offered a telephone interview or a face-to-face interview.

For face-to-face interviews, the consent form will be completed and signed as a hard copy immediately before the interview. For face-to-face interviews in which the participant is unable to provide written consent and for all telephone interviews, verbal consent will be audio recorded and taken via a standardised script immediately before the interview. In accordance with the Mental Capacity Act (2005), group 1 participants, including those identified by research nurses and assessed as fulfilling the eligibility criteria for participation, will be presumed to have capacity to consent unless it is proven otherwise.

The interviews are likely to last between 30 and $60 \mathrm{~min}$. Participants will be interviewed using a predefined topic guide (see online supplement), informed by the research aims and developed through discussion within the research team, with sufficient scope to explore novel themes where appropriate and will be audio recorded. Example PROMs will be used as visual aids. Additional data will be collected regarding patients' age, sex, ethnicity and mechanism of injury through a short demographic questionnaire. Interview recordings will be professionally transcribed using intelligent verbatim. All participants will remain anonymous, and all data will be treated as confidential except for in exceptional circumstances indicating risk, harm, malpractice or criminality.

Study dates: June 2018-December 2018.

\section{Reflectivity}

The research team comprises experts in PROMs (MC, DK, GMT, AR and AS), experienced qualitative researchers (CM, LJ, AS and AR) and a consultant neurosurgeon (TB). This qualitative study is nested within a larger programme of research aiming to develop and assess the feasibility of an ePROM pathway for inclusion within routine clinical care and TBI research.

Three researchers will conduct the interviews (CM, AR and AS). None of these researchers have any relationship with the patient, carer/relative or third sector participant groups. However, a small number of the healthcare professionals or researcher participants may be known contacts.

\section{Analysis}

Interview transcripts will be analysed using directed thematic analysis ${ }^{35}$ informed by the research aims, exploring and developing the main themes emerging 
from the data. Analysis will be undertaken simultaneously with data collection. The different participant groups will be analysed separately. A coding framework will be developed iteratively. Additional codes will be included as the analysis progresses and the framework will be modified accordingly. Formal triangulation of coding will be employed at regular intervals to enhance the credibility of the analysis. Data will be triangulated through researcher triangulation. Data will be coded and analysed by two researchers (CM and AS) who will compare and contrast the analysis and interpretation of the findings. Any differences in the analysis will be discussed until consensus has been reached. The final analysis and interpretation will also be discussed with the wider team, including our Patient and Public Involvement and Engagement (PPIE) group, to ensure that the data analysis is reliable. Differences in coding will be resolved through discussion. NVivo 11 qualitative data analysis software will be used.

\section{Patient and public involvement}

This study has been developed with consultation from members of the NIHR SRMRC Accident, Burns and Critical Care (ABC) PPIE group. This group has provided feedback on the proposed recruitment strategies, consent processes and acceptability of interview and study documents. Future points for consultation include additional recruitment strategy development; data interpretation and analyses; and dissemination of findings.

\section{ETHICS AND DISSEMINATION}

The results of this study will be disseminated via conference presentations; social media, including the Centre for Patient Reported Outcomes Research's twitter account (@CPROR_UoB), the University of Birmingham departmental website (http://www.birmingham.ac.uk/cpror); the NIHR SRMRC and peer-reviewed journals in accordance with Consolidated Criteria for Reporting Qualitative Research guidelines. ${ }^{36}$ Dissemination activities will target six key stakeholder groups:

- Patients and the public.

- Healthcare professionals/providers.

- Academics.

- NIHR SRMRC.

- Charities and third sector organisations.

- Policy-makers and commissioners.

The ABC PPIE group and stakeholder networks will be consulted to maximise dissemination and impact. ${ }^{37}$

Participants will be anonymised, and all data collected will be treated as confidential. Only anonymised, non-identifiable characteristics and quotes will be used in any arising publications or reports.

\section{DISCUSSION}

Existing evidence relating to the routine use of PROMs in clinical practice has demonstrated its utility and capacity to enrich patients' outcomes and experience of care. ${ }^{38} 39$
However, the question of the potential use of ePROMs to support people following TBI is yet to be investigated, as are their long-term impacts and support needs. Using qualitative interviews, the TBI care pathway can be established, and potential long-term impacts of TBI and commonalities in experience can be used to formulate an ePROM system that reflects patients' experiences and meets key support needs.

The main strength of our study is that we will draw on the experience of a wide range of stakeholders with the aim of capturing the most diverse possible range of perspectives to inform the development of an ePROM system. One of the limitations will be the time and resource constraints which restrict the scope of data collection. Patients with TBI will be recruited from one site (QEHB) and, therefore, the diversity of the sample will be limited by the relatively small sample. In particular, the TBI patient group will not capture the diversity of this population who are heterogeneous in demographic, clinical and injury characterises, and outcomes. Although the small sample size will reduce the transferability of our findings, this is an exploratory study and it was not our aim to achieve a representative sample. In addition, our sample may be biased by the inclusion of only participants who are assessed to have capacity to consent. As such, we will be unable to capture the perspectives and experiences of those who do not meet these criteria. However, this may be an avenue for future research.

Our exploratory work will provide valuable insight into the necessary features and functions of an ePROM system so that it is acceptable and fit for purpose for use with people with TBI. This will inform future research into the feasibility of use in routine TBI and broader trauma care. Using an e-platform to follow-up patients at different stages of the care pathway can be used to facilitate research into the impact of TBI and the unmet needs of patients and their families.

Contributors The study concept and design was conceived by GT, AS, MC, DK, LJ and TB. AR, AS and CM will consent participants and undertake the interviews with input from GT, MC, DK and LJ. The protocol was written by GT, AR prepared the first draft of the manuscript. GT, AS, CM, DK, AB, LJ and MC all provided edits and critiqued the manuscript for intellectual content.

Funding This research was funded by the National Institute for Health Research (NIHR) Surgical Reconstruction and Microbiology Research Centre (SRMRC). MC, DK and AS are partly funded by the NIHR Birmingham Biomedical Research Centre and the NIHR Surgical Reconstruction and Microbiology Research Centre at the University Hospitals Birmingham NHS Foundation Trust and the University of Birmingham. AR is employed on another project funded by Macmillan Cancer Support (grant: 5592105). DK is funded by the NIHR PDF programme (PDF2016-09-009); Macmillan Cancer Support (grant: 5592105); NIHR Birmingham Biomedical Research Centre and the NIHR Surgical Reconstruction and Microbiology Research Centre. GMT is funded by the NIHR PDR programme (PDF2017-10-047) and the NIHR SRMRC. AS is working on another project funded my Macmillan Cancer Support (grant ERN-17-0085). MC has received personal fees from Ferring and chairs the ISOQOL Best Practice for PROs in Trials Taskforce.

Disclaimer The views expressed are those of the author(s) and not necessarily those of the NHS, the NIHR or the Department of Health.

Competing interests None declared.

Patient consent for publication Not required. 
Provenance and peer review Not commissioned; externally peer reviewed.

Open access This is an open access article distributed in accordance with the Creative Commons Attribution Non Commercial (CC BY-NC 4.0) license, which permits others to distribute, remix, adapt, build upon this work non-commercially, and license their derivative works on different terms, provided the original work is properly cited, appropriate credit is given, any changes made indicated, and the use is non-commercial. See: http://creativecommons.org/licenses/by-nc/4.0/.

\section{REFERENCES}

1. The National Institute for Health and Care Excellence. Head Injury Quality Standard [QS74]. 2014. https://www.nice.org.uk/guidance/ qs74 (Accessed 1 May 2018).

2. Medical Disability Society. The management of traumatic brain injury: a working party report of the medical disability society. London: Medical Disability Society, 1988.

3. Cole TB. Global road safety crisis remedy sought: 1.2 million killed, 50 million injured annually. JAMA 2004;291:2531-2.

4. Maas Al, Stocchetti N, Bullock R. Moderate and severe traumatic brain injury in adults. Lancet Neurol 2008;7:728-41.

5. Millis SR, Rosenthal M, Novack TA, et al. Long-term neuropsychological outcome after traumatic brain injury. J Head Trauma Rehabil 2001;16:343-55.

6. Willemse-van Son AH, Ribbers GM, Verhagen AP, et al. Prognostic factors of long-term functioning and productivity after traumatic brain injury: a systematic review of prospective cohort studies. Clin Rehabil 2007;21:1024-37.

7. Schneider HJ, Kreitschmann-Andermahr I, Ghigo E, et al. Hypothalamopituitary dysfunction following traumatic brain injury and aneurysmal subarachnoid hemorrhage: a systematic review. JAMA 2007;298:1429-38.

8. Nampiaparampil DE. Prevalence of chronic pain after traumatic brain injury: a systematic review. JAMA 2008;300:711-9.

9. Gould KR, Hicks AJ, Hopwood M, et al. The lived experience of behaviours of concern: a qualitative study of men with traumatic brain injury. Neuropsychol Rehabil 2017;30(5-6):533.

10. Theadom A, Rowland V, Levack W, et al. Exploring the experience of sleep and fatigue in male and female adults over the 2 years following traumatic brain injury: a qualitative descriptive study. BMJ Open 2016;6:e010453.

11. King PR, Beehler GP, Vest BM, et al. Qualitative exploration of traumatic brain injury-related beliefs among U.S. military veterans. Rehabil Psychol 2018;63:121-30.

12. Ash JS, Cottrell E, Saxton L, et al. Patient narratives representing patient voices to inform research: a pilot qualitative study. Stud Health Technol Inform 2015;208:55-60.

13. laccarino MA, Philpotts LL, Zafonte R, et al. Stimulant use in the management of mild traumatic brain injury: a qualitative literature review. J Atten Disord 2018:108705471875975.

14. Meulenbroek P, Bowers B, Turkstra LS. Characterizing common workplace communication skills for disorders associated with traumatic brain injury: A qualitative study. J Vocat Rehabil 2016;44:15-31.

15. Fadyl JK, Theadom A, Channon A, et al. Recovery and adaptation after traumatic brain injury in New Zealand: Longitudinal qualitative findings over the first two years. Neuropsychol Rehabil 2017:1-18.

16. Graff HJ, Christensen U, Poulsen I, et al. Patient perspectives on navigating the field of traumatic brain injury rehabilitation: a qualitative thematic analysis. Disabil Rehabil 2018;40:926-34.

17. Tam S, McKay A, Sloan S, et al. The experience of challenging behaviours following severe TBI: a family perspective. Brain Inj 2015;29(7-8):813-21.

18. Carlozzi NE, Brickell TA, French LM, et al. Caring for our wounded warriors: a qualitative examination of health-related quality of life in caregivers of individuals with military-related traumatic brain injury. $J$ Rehabil Res Dev 2016;53:669-80.

19. Kratz AL, Sander AM, Brickell TA, et al. Traumatic brain injury caregivers: a qualitative analysis of spouse and parent perspectives on quality of life. Neuropsychol Rehabil 2017;27:16-37.

20. Ghajar J. Traumatic brain injury. The Lancet 2000;356:923-9.

21. Jennekens N, de Casterlé BD, Dobbels F. A systematic review of care needs of people with traumatic brain injury (TBI) on a cognitive, emotional and behavioural level. J Clin Nurs 2010;19:1198-206.

22. Dijkers MP. Quality of life after traumatic brain injury: a review of research approaches and findings. Arch Phys Med Rehabil 2004;85(4 Suppl 2):S21-35.

23. Black N. Patient reported outcome measures could help transform healthcare. BMJ 2013;346:f167.

24. Calvert MJ, Freemantle N. Use of health-related quality of life in prescribing research. Part 1: why evaluate health-related quality of life? J Clin Pharm Ther 2003;28:513-21.

25. Basch E, Jia X, Heller G, et al. Adverse symptom event reporting by patients vs clinicians: relationships with clinical outcomes. J Natl Cancer Inst 2009;101:1624-32.

26. Basch E, Deal AM, Kris MG, et al. Symptom monitoring with patientreported outcomes during routine cancer treatment: a randomized controlled trial. J Clin Oncol 2016;34:557-65.

27. Calvert M, Thwaites $R$, Kyte $D$, et al. Putting patient-reported outcomes on the 'Big Data Road Map'. J R Soc Med 2015;108:299-303.

28. Muehlhausen W, Doll H, Quadri N, et al. Equivalence of electronic and paper administration of patient-reported outcome measures: a systematic review and meta-analysis of studies conducted between 2007 and 2013. Health Qual Life Outcomes 2015;13:167.

29. Levack WM, Kayes NM, Fadyl JK. Experience of recovery and outcome following traumatic brain injury: a metasynthesis of qualitative research. Disabil Rehabil 2010;32:986-99.

30. Wilde EA, Whiteneck GG, Bogner J, et al. Recommendations for the use of common outcome measures in traumatic brain injury research. Arch Phys Med Rehabil 2010;91:1650-60.

31. Bagiella E, Novack TA, Ansel B, et al. Measuring outcome in traumatic brain injury treatment trials: recommendations from the traumatic brain injury clinical trials network. J Head Trauma Rehabil 2010;25:375-82.

32. ICF Research Branch. Development of ICF Core Sets for Traumatic Brain Injury (TBI). https://www.icf-research-branch.org/icf-core-setsprojects2/neurological-conditions/development-of-icf-core-sets-fortraumatic-brain-injury-tbi (Accessed 05 Sep 2018).

33. Honan CA, McDonald S, Tate R, et al. Outcome instruments in moderate-to-severe adult traumatic brain injury: recommendations for use in psychosocial research. Neuropsychol Rehabil 2017;2:1-21.

34. McCulloch KL, de Joya AL, Hays K, et al. Outcome measures for persons with moderate to severe traumatic brain injury: recommendations from the american physical therapy association academy of neurologic physical therapy TBI EDGE task force. $J$ Neurol Phys Ther 2016;40:269-80.

35. Fereday J, Muir-Cochrane E. Demonstrating rigor using thematic analysis: a hybrid approach of inductive and deductive coding and theme development. Int J Qual Methods 2006;5:80-92.

36. Tong A, Sainsbury P, Craig J. Consolidated criteria for reporting qualitative research (COREQ): a 32-item checklist for interviews and focus groups. Int J Qual Health Care 2007;19:349-57.

37. Cruz Rivera S, Kyte DG, Aiyegbusi OL, et al. Assessing the impact of healthcare research: a systematic review of methodological frameworks. PLoS Med 2017;14:e1002370.

38. Chen J, Ou L, Hollis SJ. A systematic review of the impact of routine collection of patient reported outcome measures on patients, providers and health organisations in an oncologic setting. BMC Health Serv Res 2013;13:211.

39. Recinos PF, Dunphy CJ, Thompson N, et al. Patient Satisfaction with Collection of Patient-Reported Outcome Measures in Routine Care. Adv Ther 2017;34:452-65. 